ARTIGOS

\title{
A IMPORTÂNCIA DAS PRÁTICAS DE MARKETING INTERNO PARA O SUCESSO ORGANIZACIONAL
}

\author{
THE IMPORTANCE OF INTERNAL MARKETING \\ PRACTICES FOR ORGANIZATIONAL SUCCESS
}

\section{RESUMO}

Este artigo tem como objetivo geral analisar se as organizações adotam práticas de marketing interno e se este contribui para o sucesso organizacional destas e de seus recursos humanos em particular. Simultaneamente, também se pretende contribuir para um melhor entendimento desse conceito, de sua aplicabilidade e consequências. Para isso, recorreu-se ao método de amostragem não probabilística por conveniência, aplicando-se 428 questionários válidos a trabalhadores em território português. Para testar as hipóteses, utilizou-se o método das equações estruturais por meio do software IBM SPSS AMOS v.25. Os resultados evidenciaram que as práticas de marketing interno promovem comprometimento afetivo, atitude de superação e satisfação no trabalho e levam a que os trabalhadores tenham uma personalidade proativa no desenvolvimento de suas funções na organização.

Palavras-chave: Marketing Interno. New Media. Recursos Humanos. Sucesso Organizacional.

\begin{abstract}
This article aims to analyze if organizations adopt internal marketing practices and whether this contributes to their organizational success and, in particular, in their human resources. Simultaneously, it is also intended to contribute to a better understanding of this concept, its applicability and consequences. To this end, we used the non-probabilistic sampling method for convenience, reaching 428 valid surveys to workers in Portuguese territory. To test the hypotheses, the structural equations method was used through the IBM SPSS AMOS v.25 software. The results showed that the internal marketing practices promote affective commitment, resourceful attitudes and job satisfaction and lead to a proactive personality on workers in the development of their functions in the organization.
\end{abstract}

Mafalda Maria Pinto Farrim Fernandes Santiago mafaldasantiago@hotmail.com Mestre em Marketing pela Faculdade de Economia da Universidade de Coimbra. Coimbra, Portugal. 
Keywords: Internal Marketing. New Media. Human Resources. Organizational Success.

\section{INTRODUÇÃO}

Atualmente, dada a volatilidade dos mercados e a intensificação da concorrência, as organizações necessitam encontrar novas formas de se diferenciarem e alcançarem vantagenm competitiva, como apostando no fator humano, tanto mais que, as "[...] estratégias e os conceitos de marketing que se focam, exclusivamente, na clientela externa já não são suficientes para alcançar os objetivos econômicos, tais como as vendas e os lucros." (STAUSS; HOFFMAN, 2000, p. 142, tradução nossa).

Assim como os consumidores, os trabalhadores devem ser considerados clientes da empresa, pois é imprescindível dar-lhes uma maior atenção, de modo que "vistam a camisola" da organização.

A área do marketing relativa à gestão eficiente e eficaz dos trabalhadores denomina-se de marketing interno, i.e., a "[...] filosofia de como tratar os trabalhadores" (SOHAIL; JANG, 2017, p. 68, tradução nossa) e surge como uma ferramenta de comunicação fundamental para a melhoria dos processos de trabalho, das relações interpessoais, da fluidez com que a informação circula na organização e na motivação, no desenvolvimento e engajamento dos trabalhadores para com ela.

Estudos prévios corroboram que a adoção de uma perspectiva de marketing interno alinha e cria relações internas, clarifica a importância de cada parte na organização e fomenta uma boa posição perante a concorrência (ABBAS; RIAZ, 2018), refletindo-se no sucesso financeiro e não financeiro organizacional (RODRIGUES; PINHO, 2012).

No entanto, apesar de, nos anos de 1990, o tema ter ganhado destaque (BOHNENBERGER; SCHMIDT; DAMACENA, 2019) e de se verificar uma maior preocupação com esse assunto, por parte dos acadêmicos e dos gestores, continua a haver uma falha na definição e implementação do conceito (MBEN-
GO; CHINAKIDZWA, 2014), muito derivado da inexistência de uma definição unificada (PARK; TRAN, 2018) e de a maioria das definições serem conceituais (HUANG; RUNDLE-THIELE; CHEN, 2018). Consequentemente, torna-se difícil compreender o que é que os marketeers, efetivamente, podem fazer (HUANG; RUNDLE-THIELE; CHEN, 2018) e como é que esse conceito decorre na prática (AHMED; RAFIQ; SAAD, 2003). Além disso, também se observa que a maioria dos estudos relativos a essa área está relacionados ao setor dos serviços (OZUEM; LIMB; LANCASTER, 2018) e tendem a focar-se em mercados tendencialmente maduros e industrializados (KADIC-MAGLAJLIE; BOSO; MICEXSKI, 2017), nomeadamente nos Estudos Unidos da América ou em Inglaterra, o que dificulta o entendimento do impacto das práticas de marketing interno nos resultados do trabalho (SOHAIL; JANG, 2017). Por fim, também se constata que a maioria dos estudos está relacionada com a orientação ao cliente, pois é necessário pesquisas relativas a todos os trabalhores e não apenas aos de contato (SOHAIL; JANG, 2017).

O principal objetivo deste estudo é analisar se as organizações adotam práticas de marketing interno e se elas contribuem para o sucesso organizacional destes e de seus recursos humanos. Para responder a esse objetivo, foi considerado um estudo cross-section por meio de um questionário, perfazendo um total de 428 respostas. Considera-se este estudo inovador, pois o modelo conceitual elaborado foi construído de raiz, recorrendo à bibliografia de referência sobre o tema, propondo-se um conjunto de novas variáveis e consequentes hipóteses que, embora já tenham sido estudadas de forma independente, nunca foram analisadas conjuntamente.

\section{DESENVOLVIMENTO CONCEITUAL E HIPÓTESES DE PESQUISA}

\subsection{MARKETING INTERNO (PMI)}

Nas últimas décadas, o conceito do 
marketing evoluiu e adaptou o seu foco central, de forma a responder às mudanças do mercado, bem como às necessidades dos consumidores.

$\mathrm{O}$ marketing interno deve ser visto como um antecedente do marketing externo (CHOI, 2016), embora as ferramentas e as técnicas que, habitualmente, são utilizadas no externo, como o marketing mix, a segmentação e a pesquisa de mercado, possam e devam ser aplicadas internamente (LINDON et al., 2004).

Esse conceito foi, inicialmente, utilizado por Berry, Hensel e Burke (1976), sob uma perspectiva direcionada para os serviços e segundo a premissa de que trabalhadores satisfeitos levariam a clientes satisfeitos, (MATZLER; FUCHS; SCHUBERT, 2004). Com o decorrer do tempo, verifica-se que "[...] uma efetiva estratégia de marketing interno vai para além dos empregados de contato" (BUSSY; EWING; PITT, 2003, p. 147, tradução nossa), deixando essa abordagem estar confinada ao referido setor (BUSSY; EWING; PITT, 2003).

Segundo Grönroos (2001), essa perspectiva do marketing considera os trabalhadores com um primeiro mercado da organização.

Rafiq e Ahmed (2000) constatam que, nos últimos anos, a literatura evidenciou a presença de três fases distintas, ainda que interligadas, para explicar o conceito.

A primeira fase é a da "Satisfação e Motivação dos Trabalhadores" e "considera os trabalhadores como clientes internos e os trabalhos como produtos internos que satisfaçam as suas necessidades e desejos, tendo presentes os objetivos da organização" (BERRY; HENSEL; BURKE, 1976, p. 8, tradução nossa), e pretende melhorar a qualidade do serviço (AHMED; RAFIQ, 2002).

A segunda fase, designada de "Orientação para o cliente", tem como objetivo motivar os trabalhadores e estimulá-los a terem uma atitude de consciência para com seus clientes (AHMED; RAFIQ, 2002).

Por último, a terceira fase, denominada "Implementação da Estratégia e Gestão das Mudanças", define o marketing interno como um processo holístico de gestão e reside em um "[...] esforço planejado para superar a resistência organizacional à mudança e alinhar, motivar e integrar os trabalhadores para a implementação efetiva de estratégias corporativas e funcionais." (RAFIQ; AHMED, 1993, p. 222, tradução nossa).

Além das três fases supracitadas, existem autores que afirmam que a orientação para os recursos humanos também contribui para uma melhor explicitação dessa abordagem (HWANG; CHI, 2005), conciliando-as com o intuito de "[...] motivar, mobilizar, cooptar e gerir os trabalhadores de todos os níveis da organização, com o objetivo de melhorar continuamente o atendimento ao cliente externo e a cada um deles." (JOSEPH, 1996, p. 55, tradução nossa).

Com o exposto, constata-se que essa perspectiva "[...] olha para os trabalhadores não como subordinados, mas segundo uma pareceria 'win-win', em que as pessoas sentem que trabalham para uma organização que lhes oferece algo em troca [...]." (GRÖNROOS, 2001, p. 332, tradução nossa).

No entanto, existem alguns problemas associados a essa prática, especificamente ao tratar os trabalhadores como clientes internos, uma vez que, e segundo Ahmed e Rafiq (2002), quando se é trabalhador, obriga-se a aceitar os produtos/serviços que a organização oferece, e a noção de trabalhador como cliente é a ideia de soberania do cliente; ora, apesar de eles serem tratados como 'clientes', não podem ter a percepção que o são efetivamente.

Importa, assim, estudar as práticas de marketing interno e suas implicações uma vez que todas as organizações enfrentam novos desafios e novos concorrentes (SOUSA, 2011), necessitando da adoção de procedimentos inovadores para conseguirem dar resposta às exigências e às necessidades de seus clientes, internos e externos, e desenvolver uma verdadeira vantagem competitiva.

Além disso, na era da modernidade líquida, em que o trabalho adquiriu um carácter mercantilizado e de curto prazo (SILVA et al., 2017) e os trabalhadores "não pretendem ape- 
nas um trabalho que garanta a sua sobrevivência, mas esperam 'gostar do que fazem', 'sentirem-se bem', 'serem desafiados', isto é, satisfazerem as suas expetativas de felicidade" (CUNHA et al., 2012, p. 100), é crucial a adoção dessa "[...] nova iniciativa cultural" (AHMED; RAFIQ, 2002, p. 63) que, antes de mais nada, 'vende' a organização a seus trabalhadores.

\subsection{NEW MEDIA USAGE (NMU)}

A comunicação é o aspecto mais tangível em um programa de marketing interno (MORGAN, 1990), sendo definida como “'[...] o conjunto de sinais emitidos pela organização em direção [...] a todos os alvos, internos e externos" (LINDON et al., 2004, p. 297, tradução nossa), com o objetivo de iniciar e construir relações, partilhar ideias, pensamentos e sentimentos, transferir informação, resolver problemas e 'conectar' as pessoas (NEILL; RICHARD, 2012).

Além dos métodos tradicionais de comunicação, que hoje já não conseguem dar resposta aos requisitos de qualidade, quantidade, velocidade e precisão (STAUSS; HOFFMAN, 2000), as novas ferramentas tecnológicas, ao dispor das organizações, constituem um 'forte aliado' no que respeita à fluidez da comunicação interna e de todas as vantagens que ela acarreta, voltando a conferir as virtudes de personalização, interatividade e da relação, como na época do marketing artesanal (LINDON et al., 2004, p. 30, tradução nossa).

Consequentemente, as organizações precisam criar e captar o valor dos new media (MUNINGER; AMMEDI; MAHR, 2019), recorrendo a capacidades estratégicas e operacionais e envolvendo os trabalhadores de vários departamentos e níveis organizacionais, pois, somente assim, conseguirão adquirir e disseminar conhecimento inovador. (MUNINGER; AMMEDI; MAHR, 2019), resolver eventuais problemas organizacionais (AHMED; RAFIQ, 2002) e sustentar uma verdadeira vantagem competitiva (GOLDEN; RAGHURAM, 2010).

Diante do exposto, propõe-se como hi- pótese de investigação:

H1: O uso dos new media tem um impacto positivo nas práticas de marketing interno.

\subsection{CONSEQUENTES DO MARKE- TING INTERNO}

O estudo do comprometimento organizacional, definido como o envolvimento dos trabalhadores para com a organização (YAO; QIU; WEI, 2019), tem ganhado destaque nas últimas décadas, pois, quando um trabalhador está comprometido com a sua organização, os resultados de seu trabalho são melhores (ALBDOUR; ALTARAWNEH, 2014).

Segundo Meyer e Allen (1991), o comprometimento organizacional engloba três componentes que caracterizam a relação entre o trabalhador e a sua organização e que têm implicações na decisão de os trabalhadores se manterem ou não nesta. São estes: (1) Comprometimento afetivo (AC), quando os trabalhadores ficam na organização porque querem; (2) Comprometimento de continuidade ou instrumental, quando os trabalhadores continuam a trabalhar na organização porque precisam; (3) Comprometimento normativo, quando os trabalhadores se sentem obrigados a permanecer na organização.

Para efeito deste estudo, apenas se terá em consideração o comprometimento afetivo, definido como o apego emocional do trabalhador, a identificação e o envolvimento para com a organização (MOHD, 2010).

Como o comprometimento organizacional dos trabalhadores é um dos resultados mais importantes do marketing interno, possibilitando a retenção de trabalhadores com capacidade e talento (ABBAS; RIAZ, 2018), propõe-se como hipótese de investigação:

H2: As práticas de marketing interno têm um impacto positivo no comprometimento afetivo organizacional.

Atualmente, as organizações regem-se pela premissa de 'fazer mais com menos', embora os gestores devam ser capazes de identificar os colaboradores que trabalham de forma 
produtiva (HARRIS et al., 2013). É nesse sentido que se torna importante o estudo da variável 'superação no trabalho' (JR).

A "superação no trabalho é definida como uma disposição duradoura para angariar recursos escassos e ultrapassar obstáculos para o alcance dos objetivos relacionados com o trabalho." (LICATA et al., 2003, p. 256, tradução nossa).

As práticas e as ferramentas de marketing interno capacitam os trabalhadores a serem mais criativos (AHMED; RAFIQ, 2002), participativos, com maior poder (XIONG; KING, 2018) e mais motivados e comprometidos (ABBAS; RIAZ, 2018), o que leva ao aumento da satisfação no trabalho e a uma melhor performance organizacional (PAUL; SAHADEV, 2016) e individual (LINGS; GREENLEY, 2005), caraterísticas presentes em um trabalhador com uma atitude de superação no trabalho. Consequentemente, propõe-se como hipótese de investigação:

H3: As práticas de marketing interno têm um impacto positivo na superação no trabalho.

Os trabalhadores são vistos como um pré-requisito indispensável para o sucesso organizacional, pelo que se revela de extrema importância o estudo da variável satisfação no trabalho (JS) (PAPASOLOMOU; VRONTIS, 2006).

Apesar de esse tema ter vindo a ser estudado ao longo dos anos por inúmeros autores, verifica-se que não existe um consenso em relação à sua definição (CRUZ; CAÑIZARES; LÓPEZ-GUZMÁN, 2011).

A definição de Locke (1969) é a mais comum para definir esse conceito, sendo aquela que vai ser considerada neste estudo, o qual a define como um estado emocional prazeroso ou positivo que resulta da avaliação da relação percebida do que se quer do trabalho em face do que se percepciona que ele oferece.

Do ponto de vista do marketing, a satisfação no trabalho tem sido analisada segundo a perspectiva de que os trabalhadores devem ser tratados com base nos princípios de satisfação baseados no cliente (ALTARIFI, 2014), o que nos remete para a adoção de uma perspectiva de marketing interno. Assim, os investigadores des- sa filosofia analisaram a satisfação no trabalho com o intuito de compreenderem como motivar e melhorar os resultados da força de trabalho (HUANG; RUNDLE-THIELE; CHEN, 2018).

Cunha et al. (2012) afirmam que a principal forma de haver trabalhadores satisfeitos é tratá-los como clientes internos e, segundo Ahmed e Rafiq (2002), essa satisfação pode ser aumentada caso se abordem as tarefas de trabalho como se tratasse de qualquer outro produto da organização, redesenhando seus componentes de acordo com aquilo que os trabalhadores valorizam. Além disso, um número considerável de estudos tem investigado o efeito do marketing interno na satisfação no trabalho (SARKER; ASHRAFI, 2018; PARK; TRAN, 2018) pelo que se propõe como hipótese de estudo:

\section{H4: As práticas de marketing interno têm um impacto positivo na satisfação no trabalho.}

As organizações de hoje dependem, cada vez mais, da proatividade de seus recursos humanos para fazer frente às mudanças que ocorrem nas estruturas e nos sistemas de trabalho (FAY; HÜTTGES, 2016).

Crant (2000) descreve quatro componentes para um melhor entendimento do conceito comportamento proativo, i.e, tendência relativamente estável para efetuar mudanças ambientais (BATEMAN; CRANT, 1993); personalidade proativa (PP), iniciativa pessoal e autoeficácia da amplitude da função e assunção de responsabilidade.

Para efeitos deste estudo, apenas se levará em consideração a personalidade proativa, mesmo que esta caracterize os trabalhadores como relativamente livres de forças situacionais e influenciadores da mudança ambiental (BATEMAN; CRANT, 1993).

As práticas e as ferramentas de marketing interno capacitam os trabalhadores a serem mais criativos (AHMED; RAFIQ, 2002), participativos, com maior poder (XIONG; KING, 2018) e a estabelecerem e melhorarem as relações de entreajuda existentes na organização por meio dos canais de comunicação disponibilizados (XIONG; KING, 2018), caraterísticas, 
também, presentes nos trabalhadores que apresentam uma personalidade proativa. Como tal, propõe-se como hipótese de estudo:

H5: As práticas de marketing interno têm um impacto positivo na personalidade proativa dos trabalhadores.

Atualmente, a maioria das pessoas passa longos períodos no trabalho, reconhecendo-o como uma parte integral da sua vida $(\mathrm{CHO}$, 2018), uma vez que as organizações devem permitir conciliar a vida pessoal com a vida profissional dos seus trabalhadores.

O bem-estar subjetivo é definido como uma experiência cognitiva na qual o indivíduo compara a sua situação atual com a situação a que aspira, espera ou sente que merece (CAMPBELL, 1976), sendo constituído por três componentes, o afeto positivo, o afeto negativo e a satisfação com a vida (SV) (ARTHAUD-DAY et al., 2005).

Para este estudo, apenas se analisará a satisfação com a vida, definida como uma avaliação global cognitiva que uma pessoa faz sobre a sua vida (DIENER et al., 1985), resultante de uma comparação entre as suas circunstâncias com um conjunto de padrões autoimpostos (DIENER, 2009).

As organizações beneficiarão caso consigam conciliar esses dois aspectos da vida dos trabalhadores, pois eles demonstrar-se-ão mais comprometidos (LAMBERT et al., 2013), mais satisfeitos com suas tarefas de trabalho (REIZER, 2015a), com uma maior predisposição para ultrapassar obstáculos, mesmo perante a escassez de recursos, e a apresentarem um maior esforço em prol da organização, realizando tarefas que vão para além dos requisitos de seus trabalhos (RHOADES; EISENBERGER, 2002). Consequentemente, propõe-se como hipóteses de estudo:

H6: O comprometimento afetivo organizacional tem um impacto positivo na satisfação com a vida.

H7: A superação no trabalho tem um impacto positivo na satisfação com a vida.

H8: A satisfação com o trabalho tem um impacto positivo na satisfação com a vida.
H9: A personalidade proativa tem um impacto positivo na satisfação com a vida.

O potencial humano é um dos principais recursos da organização, pois o grande desafio dela é conseguir reter bons trabalhadores de forma a alcançar bons resultados (DARVISH; REZAIE, 2011).

De acordo com Mott (1972), a performance (IP) de um trabalhador diz respeito à sua eficácia, sendo definida como a capacidade para mobilizar seus centros de poder para a ação. Aquela é composta por três dimensões: a produtividade, que avalia a eficiência e representa a quantidade e qualidade dos produtos/ serviços oferecidos; a adaptabilidade, que se refere à capacidade de antecipar problemas e encontrar soluções para eles; e a flexibilidade, que corresponde à capacidade dos trabalhadores em se ajustarem, rapidamente, às variações e às crises no trabalho.

Consequentemente, um indivíduo que apresente uma performance individual alta está mais comprometido para com o seu trabalho, (ALBDOUR; ALTARAWNEH, 2014), é mais propenso a ultrapassar obstáculos de forma a cumprir com seus objetivos (SEMEDO; COELHO; RIBEIRO, 2016), a estar mais satisfeito com o seu trabalho (JUDGE et al., 2001), principalmente quando se trata de um desempenho extra role (VANDENABEELE, 2009) e a prever possíveis problemas e influenciar as mudanças necessárias (THOMAS; WHITMAN; VISWESVARAN, 2010). Consequentemente, propõe-se como hipóteses de estudo:

H10: O comprometimento afetivo organizacional tem um impacto positivo na performance individual.

H11: A superação no trabalho tem um impacto positivo na performance individual.

H12: A satisfação no trabalho tem um impacto positivo na performance individual.

H13: A personalidade proativa tem um impacto positivo na performance individual.

$\mathrm{O}$ tema da criatividade (C), definida como uma ideia que leva a soluções novas e úteis (AMABILE, 1988), ganhou principal destaque nos últimos tempos, devido à intensifica- 
ção da concorrência, à globalização dos negócios e ao desenvolvimento da tecnologia, pois capacita as empresas a pensarem novas e diferenciadas formas de realizarem o trabalho, alcançando uma vantagem competitiva de longo prazo (SRIPIRABAA; MAHESWARI, 2015).

A capacidade de uma organização ser criativa depende de seus trabalhadores e de seu potencial criativo (IRIQAT, 2019), uma vez que são eles que executam o trabalho e que, por isso, têm muitas ideias de melhoria (IVANCEVICH, 2008). Consequentemente, os gestores devem incentivar seus trabalhadores a expressarem suas ideias (SOHAIL; JANG, 2017).

Um indivíduo criativo está comprometido afetivamente, pois tende a investir mais tempo no seu local de trabalho, faz sugestões de modo a resolver problemas e a encontrar soluções para eles (SEMEDO; COELHO; RIBEIRO, 2018); está mais satisfeito com seu posto de trabalho, pois se sente melhor com ele mesmo por ter mais oportunidades para ser ativo (LAMBERT; HOGAN, 2009) e é mais proativo ao procurar ativamente oportunidades e identificar novas formas de realizar o seu trabalho (KIM; HON; CRANT, 2009). Consequentemente, propõe-se como hipóteses de estudo:
H14: O comprometimento afetivo tem um impacto positivo na criatividade dos trabalhadores.

H15: A superação no trabalho tem um impacto positivo na criatividade dos trabalhadores.

H116: A satisfação no trabalho tem um impacto positivo na criatividade dos trabalhadores.

H17: A personalidade proativa tem um impacto positivo na criatividade dos trabalhadores.

\section{MÉTODO}

O modelo conceitual (figura 1) proposto apresenta o conjunto de hipóteses desenvolvidas com o intuito de responder aos objetivos do estudo. Para isso, concebeu-se um estudo cross-section por meio de um questionário, obtendo-se um total de 428 respostas de trabalhadores em território nacional, alcançadas pelo método de amostragem não probabilística por conveniência. Para testar as 17 hipóteses, recorreu-se ao modelo das equações estruturais no software estatístico IBM SPSS AMOS v.25.

Figura 1 - Modelo Conceptual

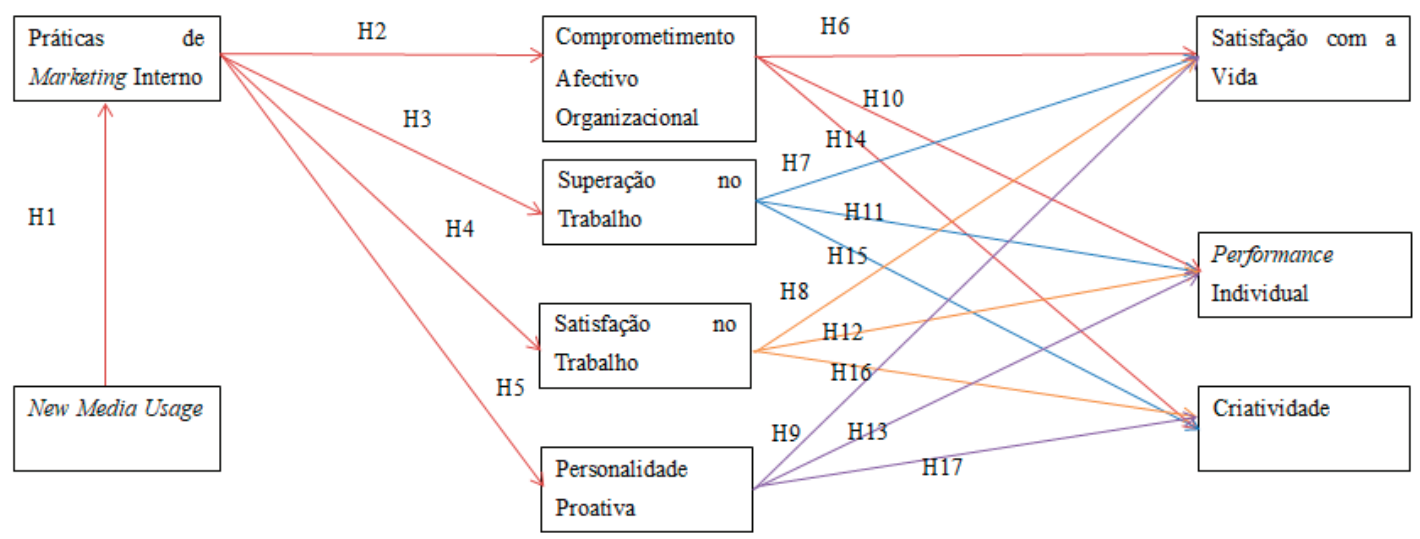

Fonte: elaboração própria (2019).

\section{ANÁLISE ESTATÍSTICA}

4.1 CARATERIZAÇÃO DA AMOSTRA

A amostra é constituída por $51,2 \%$ de mulheres e $48,8 \%$ de homens, embora $74,1 \%$ dos participantes tenham completado o ensino superior. Em relação à idade, esta varia entre os 18 e os 68 anos. Constata-se, assim, que os participantes se situam, essencialmente, entre 
os 26 e os 55 anos $(69,4 \%)$, o que pode ser justificável pelo fato de o questionário ter sido administrado via online. A grande parte dos participantes é trabalhadora por causa de outrem $(79,7 \%)$, de primeira ordem $(58,4 \%)$ e exerce funções nas respetivas organizações há menos de cinco anos (50,7\%). Também é de salientar que $36,7 \%$ das organizações têm mais de 250 funcionários, sendo as restantes PME's, e que $15,2 \%$ dos inquiridos trabalham nas respetivas organizações há menos de 10 anos, e 84,8\%, há mais de 10 anos. Por fim, no que se refere ao rendimento do agregado familiar, verifica-se que ele está concentrado nas classes dos 500 aos $999 €(23,1 \%), 1000-1499 €(25 \%)$ e 1500 a $2499 €(26,4 \%)$.

\subsection{MÉTRICAS}

O questionário é constituído por questões fechadas e, para a medição das métricas, recorreu-se à Escala de Likert de 7 pontos (1- discordo fortemente; 7- concordo fortemente), em que é pedido aos participantes para indicarem o grau de concordância relativamente aos itens utilizados; com exceção da variável new media usage, em que foi acrescentada uma opção de não resposta, pois as organizações podem não utilizar a internet, intranet e/ou e-mail para comunicarem internamente e da performance individual, em que o 1 é referente a 'muito abaixo da média' e o 7 a 'muito acima da média'.

As práticas de marketing interno foram medidas utilizando a escala de Huang e Rundle-Thiele (2014), constituída por três dimensões, de forma a compreender como é que os trabalhadores percepcionam a comunicação interna, as ofertas de formação e a realização de pesquisa de mercado interna dentro das respetivas organizações. Para a mediação da new media usage, recorreu-se à escala desenvolvida por Bussy, Ewing e Pitt (2003), composta por três dimensões, com o intuito de compreender se as organizações utilizam a internet, intranet e/ou e-mail, para comunicarem internamente. A métrica do comprometimento afetivo organizacional foi desenvolvida por Meyer, Allen e Smith (1993) e tem como objetivo perceber se os trabalhadores estão envolvidos para com a sua organização. Relativamente à superação no trabalho, recorreu-se à métrica de Licata et al. (2003), de modo a entender se os inquiridos estão dispostos a fazer um esforço adicional em prol de sua organização. A métrica da satisfação no trabalho é composta pela escala Huang e Rundle-Thiele (2014) e dois itens desenvolvidos por Brayfield e Rothe (1951) e tem como objetivo compreender se os trabalhadores estão satisfeitos e entusiasmados com as oportunidades que o seu trabalho lhes proporciona. No que se refere à personalidade proativa, foi utilizada a escala de Bateman e Crant (1993) de forma a compreender se os trabalhadores têm iniciativa própria para alcançar as metas pré-estabelecidas e se são capazes de identificar oportunidades de melhoria. A satisfação com a vida foi medida recorrendo à escala desenvolvida por Diener et al., (1985) e tem como objetivo compreender se os inquiridos estão satisfeitos com aquilo que alcançaram até ao momento. A métrica performance individual desenvolvida por Mott (1972) é composta por três dimensões: flexibilidade, adaptabilidade e produtividade, e tem como propósito compreender como é que os trabalhadores se posicionam relativamente a elas. Por fim, a criatividade foi desenvolvida por Zhou e George (2001) e pretende compreender se os trabalhadores têm e usam novas ideias e práticas para a execução de seu trabalho.

Assim, as métricas utilizadas foram traduzidas da língua inglesa para a portuguesa e foram testadas por meio da realização de um pré-teste a 30 trabalhadores representativos da população com o intuito de testar a fidedignidade do instrumento.

\subsection{VALIDADE}

Todos os itens foram medidos por meio de uma escala de Likert de 7 pontos. A análise fatorial confirmatória foi utilizada para avaliar a qualidade das escalas bem como o modelo de medidas, recorrendo-se o software estatístico IBM SPSS AMOS v.25. 
O modelo de medidas final é revelador de um bom fit (Incremetal fit índex $=0,954$; Tucker-Lewis índex $=0,952$; comparative fit index $=0,954$; root mean square error of approximation $=0,039 ; \mathrm{X} 2 / \mathrm{DF}=1,665)$. -se a análise do modelo estrutural, com o intuito de testar as hipóteses propostas. O modelo final é revelador de um bom fit (Incremetal fit índex = 0,937; Tucker-Lewis índex $=0,933$; comparative fit index $=0,936$; root mean square error of appro-

Tabela 1 - Desvio-padrão (DP), matriz de correlações, Alpha de Cronbach (sublinhado), Variância Média Extraída (AVE) e Fiabilidade Compósita (CR)

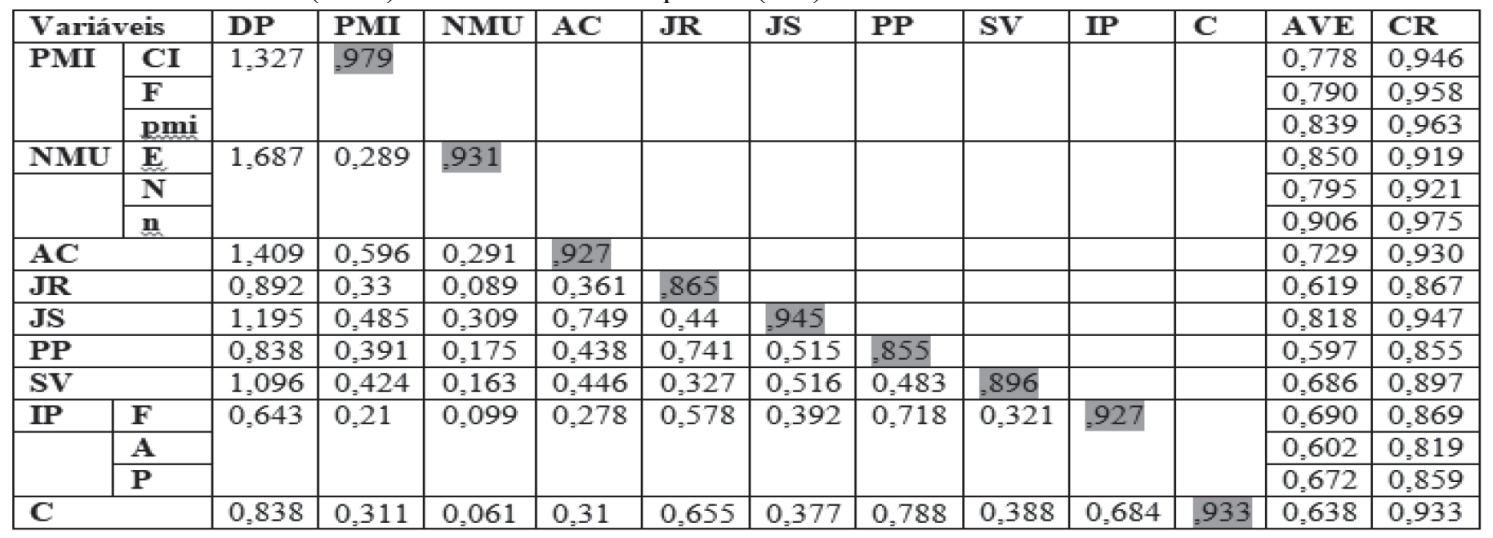

Nota: CI- comunicação interna; F- formação; pmi-pesquisa de mercado interno; E- email; N- internet; nintranet; F- flexibilidade; A- adaptabilidade; P-produtividade

Fonte: elaboração própria (2019).

Para a análise da fiabilidade das variáveis, analisou-se o CR (Composite reliability), constatando-se que todos os valores são superiores a 0,7 , o que está de acordo com as recomendações da literatura (HAIR et al., 2014), e o AVE (average variance extracted), que apresenta resultados superiores a 0,5, aceitando-se, assim, o pressuposto da fiabilidade. (HAIR et al., 2014). Relativamente à validade discriminante, observa-se que as correlações entre as variáveis são inferiores à AVE, o que se conclui pela validade discriminante, com exceção da correlação entre a performance individual e a criatividade. No entanto, e seguindo as indicações de Fornell e Larcker (1981), após redesenhar-se o modelo apenas com essas duas variáveis e fixando a correlação em 1 , verifica-se que ele é melhor quando esta não está fixa em 1, concluindo-se pela validade discriminante (tabela 1).

\section{RESULTADOS}

Após garantir-se que o modelo de medidas é fiável e apresenta um bom ajustamento, segue- ximation $=0,046 ; \mathrm{X} 2 / \mathrm{DF}=1,913)$.

A tabela 2 apresenta os resultados finais da amostra. Todas as hipóteses foram testadas recorrendo ao modelo das equações estruturais.

Tabela 2 - Teste de Hipóteses

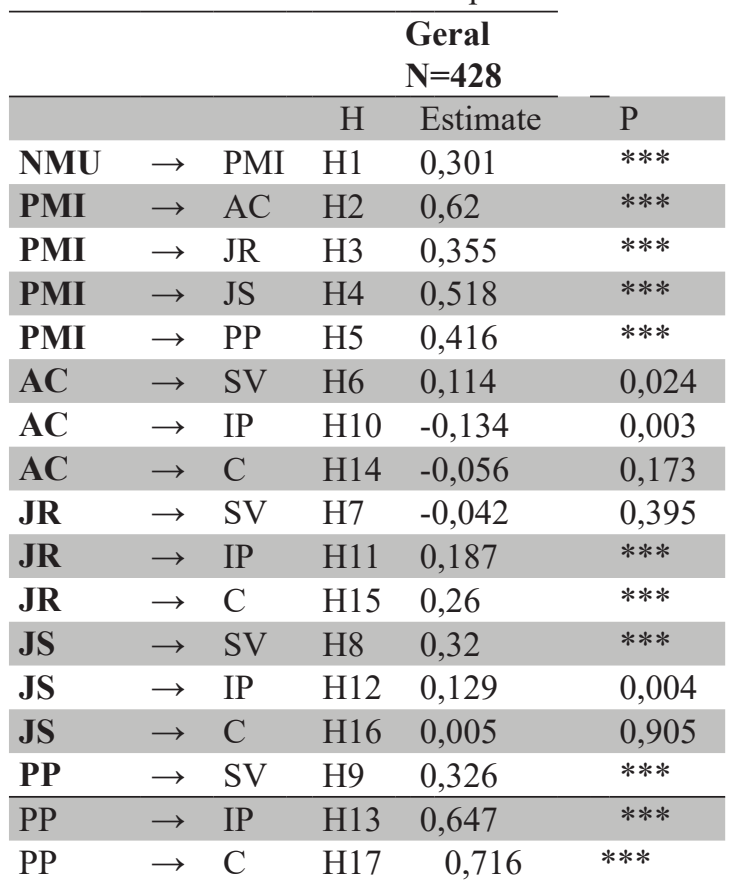


De acordo com o exposto por Muninger, Hammedi e Mahr (2019), conclui-se que o uso dos new media tem um impacto positivo nas práticas de marketing interno $(b=0,301 ; p<0,01), o$ que suporta a H1. As organizações que criam e capturam valor dos new media, envolvendo todos os trabalhadores, estão mais propensas a adquirir e a disseminar conhecimento inovador (MUNINGER; HAMMEDI; MAHR, 2019) e a alcançar uma verdadeira vantagem competitiva (GOLDEN; RAGHURAM, 2010).

No que diz respeito às práticas de marketing interno, encontrou-se suporte estatístico para confirmar sua contribuição positiva para o comprometimento afetivo organizacional $(b=0,62 ; p<0,01)$, a superação no trabalho $(b=0,355 ; p<0,01)$, a satisfação no trabalho $(b=0,518 ; p<0,01)$ e para a personalidade proativa $(b=0,416 ; p<0,001)$, o que suporta a H2, H3, H4 e H5. O recurso por parte das organizações ao marketing interno pode levar os trabalhadores a estar mais comprometidos afetivamente, o que leva à sua retenção com capacidade e talento (ABBAS; RIAZ, 2018), e que estejam mais propensos a ultrapassar possíveis obstáculos e a empenhar um esforço extra, mesmo perante a escassez de recursos (LICATA et al., 2003), a estarem mais entusiasmados e prazerosos com seu trabalho (PARK; TRAN, 2018) e com uma predisposição de forte iniciativa para alcançarem as metas desejadas (AKGUNDUZ; ALKAN; GÖK, 2018).

Relativamente ao comprometimento afetivo organizacional, observa-se que, essa variável tem um impacto positivo na satisfação com $a$ vida $(b=0,114 ; p<0,05)$, um impacto negativo com a performance individual $(b=-0,134$; $\mathrm{p}<0,01)$ e não tem impacto na criatividade $(\mathrm{b}=$ $0,056 ; p>0,1)$. Consequentemente, constata-se que apenas a relação entre o comprometimento afetivo organizacional e a satisfação com a vida (H6) foi prevista na literatura (LAMBERT et al., 2013). Essa constatação pode dever-se ao fato de, e tal como afirmam Meyer, Allen e Smith (1993), os trabalhadores que têm uma experiência organizacional consistente com as suas expetativas tendem a desenvolver um apego emocional e uma grande identificação e envolvimento para com a sua organização. No que se refere à ligação com a performance individual (H10), Joelle e Coelho (2017, p. 18, tradução nossa) explicam esta relação negativa afirmando que "[...] quando os trabalhadores estão muito comprometidos com a organização tendem a menosprezar o seu desempenho e a superestimar as suas obrigações, centrando os seus esforços numa melhoria contínua da sua performance." Por fim, apesar de Semedo, Coelho e Ribeiro (2018) e Tenzer e Yang (2018) verificarem que o comprometimento afetivo está relacionado com a criatividade, neste estudo, essa hipótese (H14) não foi corroborada; possível explicação para tal pode dever-se ao fato de os trabalhadores não terem abertura nem espaço para fazerem sugestões de novas ideias e/ou de melhorias, ou pelo cargo, ou pelo tipo de organização, ou porque estes não estabeleceram um verdadeiro laço emotivo com a organização na qual trabalham.

Analisando a variável superação no trabalho, observa-se que esta não tem impacto na satisfação com a vida e tem um impacto positivo na performance individual $(b=0,187$; $\mathrm{p}<0,01)$ e na criatividade $(b=0,26 ; p<0,01)$. Assim, apenas essas duas últimas relações são suportadas na literatura. Apesar de Vitorino e Coelho (2018) terem decidido por uma relação entre a superação no trabalho e a satisfação com a vida, neste estudo, os resultados não corroboraram (H7); esse fato pode ser justificável porque os indivíduos que têm uma atitude de superação no trabalho têm que empenhar um maior esforço em suas tarefas de trabalho, dedicando mais tempo à organização, o que pode dificultar a conciliação entre a vida profissional e a pessoal. No que se refere à relação com a performance individual (H11), observa-se que os resultados alcançados seguem a mesma linha de pensamento de Joelle e Coelho (2017); i.e., verifica-se que os trabalhadores que revelam um maior nível de superação no trabalho consideram-se os mais produtivos e com uma performance superior (HARRIS et al., 2006). Por último, Cheng e Chen (2016) e Semedo, 
Coelho e Ribeiro (2018) também verificaram uma relação positiva entre a superação no trabalho e a criatividade (H15); isso pode dever-se ao fato de os indivíduos com uma predisposição resourceful apresentarem uma motivação interna que os leva a arranjar ideias novas e úteis.

No que se refere à variável satisfação no trabalho, observa-se que esta tem um impacto positivo na satisfação com a vida $(b=0,32 ; p<0,01)$ e na performance individual $(b=0,219 ; p<0,01)$, o que corrobora o descrito na literatura. No entanto, não se corrobora a relação entre a satisfação no trabalho e a criatividade $(\mathrm{H} 16)(\mathrm{b}=0,005$; $\mathrm{p}>0,1$ ), o que pode ser explicado pelo fato de os trabalhadores, ao estarem satisfeitos com seu trabalho, já não terem necessidade de ir mais além, não aplicando um maior esforço em suas tarefas de trabalho. Em relação à satisfação com a vida (H8), esta foi corroborada por Reizer (2015b) e pode ser justificada pelo fato de, para a maioria das pessoas, o trabalho é um aspecto central de suas vidas. Por fim, Haryono, Ambarwati e Saad (2019) também sustentam a relação com a performance individual (H12), pois trabalhadores satisfeitos tendem a empenhar um maior esforço em seu trabalho (SIBHOKO; BAYAT, 2019).

Relativamente à personalidade proativa, constata-se que esta tem um impacto positivo na satisfação com a vida $(b=0,326 ; p<0,01)$, na performance individual $(b=0,647 ; p<0,01)$ e na criatividade $(b=0,716 ; p<0,01)$, tal como previsto na literatura $(\mathrm{H} 9, \mathrm{H} 13$ e $\mathrm{H} 17$, respetivamente). Os trabalhadores que têm uma personalidade proativa, ao sentirem que a organização se preocupa com eles, estão mais propensos a esforçarem-se, apresentando um comportamento extrapapel (RHOADES; EISENBERGER, 2002), a apresentarem uma maior performance no trabalho em geral e nas suas tarefas em particular (SPITZMULLER et al., 2015) e a procurarem ativamente oportunidades, a fim de identificarem novas formas de realizar o seu trabalho (KIM; HON; CRANT, 2009).

\section{CONCLUSÃO}

O objetivo central deste estudo é com- preender se a adopção por parte das organizações, das práticas de marketing interno, contribuem, ou não, para o sucesso organizacional no seu todo e dos seus recursos humanos em particular. Para responder a esse objetivo, foi realizado um estudo cross-section, baseado em um questionário com 428 respostas. De modo a testar as inúmeras hipóteses, recorreu-se ao modelo das equações estruturais.

Os resultados deste estudo demonstraram que o recurso aos new media são cruciais para criar e capturar valor para as organizações e que as práticas de marketing interno têm impacto no comprometimento afetivo, na superação no trabalho, no aumento da performance individual bem como na personalidade proativa.

Essas constatações podem ser úteis para que as organizações entendam que, para alcançarem uma verdadeira vantagem competitiva, precisam apostar em seus recursos intangíveis. Os resultados, também, devem estimular novas pesquisas na área do marketing interno, pois este estudo apresenta novas e interessantes relações que podem ser desenvolvidas e testadas em outras investigações.

\section{CONTRIBUIÇÕES E LIMITAÇÕES}

\subsection{CONTRIBUIÇÕES TEÓRICAS}

$\mathrm{O}$ marketing interno surge como uma ferramenta de comunicação fundamental para a melhoria dos processos de trabalho, das relações interpessoais, da fluidez com que a informação circula na organização e motivação, no desenvolvimento e engajamento dos trabalhadores para com ela. Assim, é necessário que haja uma clarificação do conceito (PARK; TRAN, 2018) para que os profissionais de marketing compreendam em que é que consiste efetivamente o marketing interno (HUANG; RUNDLE-THIELE; CHEN, 2018) e como é que ele decorre na prática (AHMED; RAFIQ; SAAD, 2003).

Este estudo é inovador, apresentando, essencialmente, três grandes contribuições: 
(1) parte de definições tradicionais para uma aplicação empírica, mostrando os efeitos do marketing interno; (2) operacionaliza o conceito identificando práticas e modos de ação do marketing interno; (3) mostra como as práticas de marketing interno podem influenciar os resultados em nível individual, dos recursos humanos e organizacional.

\subsection{CONTRIBUIÇÕES PRÁTICAS}

O marketing interno parte do pressuposto de que os trabalhadores são o primeiro mercado da organização (GRÖNROOS, 2001); como tal, não pode ser negligenciado em um mundo em constante mudança e, cada vez mais, complexo, pois ajuda a alinhar e a criar relações internas (ABBAS; RIAZ, 2018) e a melhorar os processos de trabalho.

Essa investigação poderá ajudar na elaboração de planos de marketing ao mostrar que o marketing interno pode ser uma ferramenta imprescindível para solidificar a relação entre todos os recursos humanos que constituem a empresa, ao conhecer as suas necessidades e expetativas e ao melhorar os processos de trabalho, o que, por sua vez, terá repercussões em nível de seu sucesso organizacional.

\subsection{LIMITAÇÕES E PESQUISAS FU- TURAS}

Esta investigação é baseada em um estudo cross-section de uma amostra de 428 trabalhadores portugueses. Em estudos futuros, dever-se-ia considerar uma amostra superior, alcançada por meio de um método de amostragem probabilístico de forma a conseguir extrapolar os dados para a população. Também se poderia conjugar com outras metodologias de pesquisa para fazer uma comparação entre o setor privado e o setor público português.

Além do referido, e no que diz respeito às futuras linhas de investigação, identifica-se a necessidade da realização de mais estudos nesta área do marketing.

\section{REFERÊNCIAS}

ABBAS, A.; RIAZ, M. The effect of internal marketing dimensions on organizational commitment of employees: An Investigation among Private Banks in Faisalabad. European online Journal of Natural and Scocial Sciences, Pakistan, v. 7, n. 1, p. 147-165, jan. 2018. Disponível em: http://www.european-science. com. Acesso em: 17 jan. 2019.

AHMED, P.; RAFIQ, M. Internal Marketingtools and concepts for customer-focused management. Woburn: Butterworth-Heinemann, 2002.

AHMED, P.; RAFIQ, M.; SAAD, N. Internal marketing and the mediating role of organisational competencies. European Journal of Marketing, v. 37, n. 9, p. 1221-1241, 2003.

AKGUNDUZ, Y.; ALKAN, C.; GÖK, Ö. A. Perceived organizational support, employee creativity and proactive personality: The mediating effect of meaning of work. Journal of Hospitality and Tourism Management, Turkey, v. 34, p. 105-114, jan. 2018.

ALBDOUR, A. A.; ALTARAWNEH, I. I. Employee engagement and organizational commitment: evidence from Jordan. International Journal of Business, v. 19, n. 2, 2014.

ALTARIFI, S. Internal Marketing Activities in Higher Education. International Journal of Business and Management, Canada, v. 9, n. 6, p. 126-138, maio 2014.

AMABILE, T. M. A model of creativity and innovation in organizations. Research in Organizational Behavior, v. 10, p. 123-167, 1988.

ARTHAUD-DAY, M. L. et al. The subjective well-being construct: a test of its convergent, discriminant, and fatorial validity. Social Indicators Research, v. 74, 2005. 
BATEMAN, T.; CRANT, J. M. The proactive component of organizational behavior: a measure and correlates. Journal of Organizational Behavior, v. 14, p. 103-118, 1993.

BERRY, L.; HENSEL, J.; BURKE, M. Improving retailer capability for effective consumerism response. Journal of Retailing, v. 52, n. 3, p. 3-13, 1976.

BOHNENBERGER, M. C.; SCHMIDT, S.; DAMACENA, C., Internal Marketing: A Model for Implementation and Development. Dimensión Empresarial, v. 17, n. 1, p. 7-22, 2019.

BRAYFIELD, A. H.; ROTHE, H. F. An index of job satisfaction. Journal of Applied Psychology, v. 35, n. 5, p. 307-311, 1951.

BUSSY, N. de; EWING, M.; PITT, L. Stakeholder theory and internal marketing communications: A framework for analysing the influence of new media. Journal of Marketing Communications, v. 9, n. 3, p. 147-161, 2003.

CAMPBELL, A. Subjective measures of well-being. American psychologist, Chicago, p. 117-124, 1976.

CHENG, J. C.; CHEN, C. Y. Job resourcefulness, work engagement and prosocial service behaviors in the hospitality industry. International Journal of Contemporary Hospitality Management, v. 29, n. 10, p. 1-39, 2016.

CHO, S. Effects of social support and grateful disposition on employees' psychological well-being. The Service Industries Journal, p. 1-21, 2018.

CHOI, S. An Inside-Out Marketing Strategy for Innovation among Human Service Nonprofits in South Korea. Nonprofit Management \& Leadership, v. 26, n. 3, p. 331-347, 2016.

CRANT, J. M. Proactive Behavior in Organizations. Journal of Management, v. 26, n. 3, p. 435-462, 2000.
CRUZ, F.; CAÑIZARES, S.; LÓPEZ-GUZMÁN, T. Satisfacción laboral como fator crítico para la calidad: El caso del sector hostelero de la provincia de Córdoba - España. Revista Estudios y Perspectivas en Turismo, v. 20, p. 1047-1068, 2011.

CUNHA, M. et al. Manual de Gestão de Pessoas e do Capital Humano. 2. ed. Lisboa: Sílabo, 2012.

DARVISH, H.; REZAIE, F. The impact of authentic leadership on job satisfaction and team commitment. Management \& Marketing, v. 6, n. 3, p. 421-436, 2011.

DIENER, E. Assessing Well-Being. USA: Springer, 2009.

DIENER, E. et al. The Satisfaction With Life Scale. Journal of Personality Assessment, v. 49, n. 1, p. 71-75, 1985.

FAY, D.; HÜTTGES, A. Drawbacks of Proactivity: Effects of Daily Proactivity on Daily Salivary Cortisol and Subjective Well-Being. Journal of Occupational Health Psychology, p. 1-14, 2016.

FORNELL, C.; LARCKER, D. Evaluating structural equation models with unobservable variables and measurement error: a comment. Journal of Marketing Research, v. 18, n. 3, p. 39-50, 1981.

GOLDEN, T.; RAGHURAM, S. Teleworker knowledge sharing and the role of altered relational and technological interactions. Journal of Organizational Behavior, v. 31, 2010.

GRÖNROOS, C. Service Management and Marketing. 2. ed. England: John Wiley \& sons, 2001.

HAIR, J. et al. Multivariate data analysis. 7. ed. Harlow: Pearson Education Limited, 2014. 
HARRIS, E. G. et al. Role stressors, service worker job resourcefulness, and job outcomes: An empirical analysis. Journal of Business Research, v. 59, p. 407-415, 2006.

HARRIS, E. G. et al. Examining the Influence of Job Resourcefulness on Sales Performance. Journal of Marketing Theory and Practice, v. 21, n. 4 , p. $405-414,2013$.

HARYONO, S.; AMBARWATI, Y. I.; SAAD, M. S. M. Do Organizational Climate and Organizational Justice Enhance Job Performance Through Job Satisfaction ? a Study of Indonesian Employees. Academy of Strategic Management Journal, v. 18, n. 1, p. 1-6, 2019.

HUANG, Y. T.; RUNDLE-THIELE, S. The moderating effect of cultural congruence on the internal marketing practice and employee satisfaction relationship: an empirical examination of Australian and Taiwanese born tourism employees. Tourism Management, v. 42, p. 196-206, 2014.

HUANG, Y. T.; RUNDLE-THIELE, S.; CHEN, Y. H. Extending understanding of the internal marketing practice and employee satisfaction relationship: a bugget chinese airline empirical examination. Journal of Vacation Marketing, v. 20, n. 10, p. 1-11, 2018.

HWANG, I. S.; CHI, D. J. Relationships among Internal Marketing, Employee Job Satisfaction and International Hotel Performance: An Empirical Study. International Journal of Management, v. 22, n. 2, p. 285-293, 2005.

IRIQAT, R. A. M. The Effect of environment supports on individual creativity in palestinian small and medium enterprises: assessing the mediating role of general self - efficacy. International Review of Management and Marketing, v. 9, n. 1, p. 129-136, 2019.

IVANCEVICH, J. Gestão de recursos humanos. 10. ed. São Paulo: McGraw-Hill Interamericana do Brasil Lda, 2008.
JOELLE, M.; COELHO, A. M. The impact of spirituality at work on workers' attitudes and individual performance. The International Journal of Human Resource Management, p. 1-25, 2017.

JUDGE, T. A. et al. The job satisfaction-job performance relationship: a qualitative and quantitative review. Psychological Bulletin, v. 127, n. 3, p. 376-407, 2001.

KIM, T. Y.; HON, A.; CRANT, J. M. Proactive personality, employee creativity, and newcomer outcomes: a longitudinal study. Journal of Business and Psychology, v. 24, p. 93-103, 2009.

LAMBERT, E. G.; HOGAN, N. L. Wanting change: the relationship of perceptions of organizational innovation with correctional staff job stress, job satisfaction, and organizational commitment. Criminal Justice Police Review, v. 20, n. 10, p. 1-25, 2009.

LAMBERT, E. G. et al. The association of affective and continuance commitment with correctional staff life satisfaction. Social Science Journal, v. 50, p. 195-203, 2013.

LICATA, J. W. et al. On the trait atecedents and outcomes of service worker job resourcefulness: A Hierarchical Model Approach. Journal of the Academy of Marketing Science, v. 31, p. 256-271, 2003.

LINDON, D. et al. Mercator XXI Teoria e prática do marketing. 10. ed. Lisboa: Dom Quixote, 2004.

LINGS, I. N.; GREENLEY, G. E. Measuring Internal Market Orientation. Journal of Service Research, v. 7, n. 3, p. 290-305, 2005.

LOCKE, E. A. What is Job Satisfaction? Organizational Behavior and Human Performance, v. 4, p. 309-336, 1969.

MATZLER, K.; FUCHS, M.; SCHUBERT, A. 
Employee satisfaction: Does Kano's model apply? Total Quality Management and Business Excellence, v. 15, n. 9/10, p. 1179-1198, 2004.

MBENGO, P.; CHINAKIDZWA, M., Internal Marketing Elements' Influence on Employee Performance : A Case of Harare Institute of Technology in Zimbabwe. Journal of Business Administration and Education, v. 5, n. 2, p. 191-207, 2014.

MEYER, J. P.; ALLEN, N. J. A three-component conceptualization of organizational commitment. Human Resource Management Review, v. 1, n. 1, p. 61-89, 1991.

MEYER, J. P.; ALLEN, N. J.; SMITH, C. A. Commitment to Organizations and Occupations: Extension and Test of a Three-Component Conceptualization. Journal of Applied Psychology, v. 78, n. 4, p. 538-551, 1993.

MOHD, H. J. Organizational Commitment and Employee's Innovative Behavior - A Study in Retail Sector. Journal of Management Research, v. 10, n. 1, p. 62-68, 2010.

MORGAN, N. A. Implementing marketing: key issues for professional service firms. Journal of Professional Services Marketing, v. 6, n. 1, p. 7-16, 1990.

MOTT, P. The Characteristics of Effective Organizations. New York: Harper \& Row, 1972.

MUNINGER, M. I.; HAMMEDI, W.; MAHR, D. The value of social media for innovation: A capability perspective. Journal of Business Research, v. 95, 116-127, 2019.

NEILL, W.; RICHARD, J. Intranet portals: marketing and managing individuals' acceptance and use. Australasian Marketing Journal, v. 20, p. 147-157, 2012.

OZUEM, W.; LIMB, N.; LANCASTER, G.
Exploring the locus of internal marketing. Journal of Strategic Marketing, v. 26, n. 4, p. 356-372, 2018.

PAPASOLOMOU, I.; VRONTIS, D., Building corporate branding through internal marketing: the case of the UK retail bank industry. Journal of Product \& Brand Management, v. 15, n. 1, p. 37-47, 2006.

PARK, J. H.; TRAN, T. B. H. Internal marketing, employee customer-oriented behaviors, and customer behavioral responses. Psychology and Marketing, v. 35, n. 6, p. 412-426, 2018.

PAUL, J.; SAHADEV, S. Service failure and problems: Internal marketing solutions for facing the future. Journal of Retailing and Consumer Services, p. 1-8, 2016.

RAFIQ, M.; AHMED, P. The Scope of Internal Marketing: Defining the Boundary Between Marketing and Human Resource Management. Journal of Marketing Management, v. 9, p. 219-232, 1993.

RAFIQ, M.; AHMED, P. Advances in the internal marketing concept: definition, synthesis and extension. Journal of services marketing, v. 14, n. 6, p. 449-462, 2000.

REIZER, A. Influence of employees attachment styles on their life satisfaction as mediated by job satisfaction and burnout. Journal of Psychology: Interdisciplinary and Applied, v. 149 , n. 4 , p. $356-377,2015 a$.

REIZER, A. Influence of employees attachment styles on their life satisfaction as mediated by job satisfaction and burnout. Journal of Psychology: Interdisciplinary and Applied, v. 149, n. 4, p. 356-377, 2015 b.

RHOADES, L.; EISENBERGER, R. Perceived organizational support: a review of the literature. Journal of Applied Psychology, v. 87, n. 4, p. 698-714, 2002. 
RODRIGUES, A.; PINHO, J. The impact of internal and external market orientation on performance in local public organisations. Marketing Intelligence \& Planning, v. 30, n. 3, p. 284-306, 2012.

SARKER, A. R.; ASHRAFI, D. M. The relationship between internal marketing and employee job satisfaction: A study from retail shops in Bangladesh. Journal of Business and Retail Management Research, v. 12, n. 3, p. 149-159, 2018.

SEMEDO, A.; COELHO, A.; RIBEIRO, N. Effects of authentic leadership, affective commitment and job resourcefulness on employees' creativity and individual performance. Leadership \& Organization Development Journal, v. 37, n. 8, p. 1-21, 2016.

SEMEDO, A.; COELHO, A.; RIBEIRO, N. The relationship between authentic leaders and employees' creativity: What are the roles of affective commitment and job resourcefulness? International Journal of Workplace Health Management, p. 58-73, 2018.

SIBHOKO, O.; BAYAT, M. S. An investigation into employee job satisfaction and its impact on organizational effectiveness with special reference to the buffalo city college. Management Studies and Economic Systems, v. 4, n. 1, p. 71-78, 2019.

SILVA, L. et al. Sobre as relações de trabalho na modernidade líquida: reflexões a partir de Zygmunt Bauman. Revista Brasileira de Educação e Cultura, v. 16, n. 4, p. 45-56, 2017.

SOHAIL, M. S.; JANG, J. Understanding the relationships among internal marketing practices, job satisfaction, service quality and customer satisfaction: an empirical investigation of Saudi Arabia's service employees. International Journal of Tourism Sciences, v. 17, n. 2, p. $67-85,2017$.
SOUSA, A. Orientação do Marketing no Sector Público- O marketing-mix da iniciativa "Novas Oportunidades”. Economia \& Empresa, v. 13, p. 49-72, 2011.

SPITZMULLER, M. et al. Investigating the uniqueness and usefulness of proactive personality in organizational research: a meta-analytic review. Human Performance, v. 28, n. 4, p. 351-379, 2015.

SRIPIRABAA, B.; MAHESWARI, S. Individual creativity: influence of job autonomy and willingness to take risk. Journal of Indian Management, p. 110-119, 2015.

STAUSS, B.; HOFFMAN, F., Minimizing internal communication gaps by using business television, In: VAREY, Richard J.; LEWIS, Barbara; LEWIS, Barbara R. Internal Marketing: Directions for Management. Londres: [s.n.], 2000. p. 141-159.

TENZER, H.; YANG, P. Personality, Values, or Attitudes? Individual-Level Antecedents To Creative Deviance. International Journal of Innovation Management, v. 23, n. 2, 1950009, 2018.

THOMAS, J. P.; WHITMAN, D. S.; VISWESVARAN, C. Employee proactivity in organizations: a comparative meta-analysis of emergent proactive constructs. Journal of Occupational and Organizational Psychology, v. 83, p. 275300, 2010.

VANDENABEELE, W. The mediating effect of job satisfaction and organizational commitment on self-reported performance: more robust evidence of the PSM - Performance relationship. International Review of Administrative Sciences, v. 75, n. 1, p. 11-34, 2009.

VITORINO, P.; COELHO, A. Antecedentes e consequentes da satisfação com o voluntariado empresarial: um estudo empírico. 2018. Dissertação (Mestrado em Marketing) - Facu- 
dade de Economia, Universidade de Coimbra, 2018.

XIONG, L.; KING, C. Too much of a good thing? Examining how proactive personality affects employee brand performance under formal and informal organizational support. International Journal of Hospitality Management, v. 68, p. 12-22, 2018.

YAO, T.; QIU, Q.; WEI, Y. Retaining hotel employees as internal customers: effect of organizational commitment on attitudinal and behavioral loyalty of employees. International Journal of Hospitality Management, v. 76, p. 1-8, 2019.

ZHOU, J.; GEORGE, J. M. When job dissatisfaction leads to creativity: encouraging the expression of voice. Academy of Management Journal, v. 44, n. 4, p. 682-696, 2001. 